\title{
CALCULATION OF $J$-INTEGRAL FOR LARGE STRAINS USING THE FINITE ELEMENT METHOD
}

\author{
ÁgNes HoRváth \\ Institute of Applied Mechanics, \\ University of Miskolc, H-3515 Miskolc-Egyetemváros \\ mechva@uni-miskolc.hu
}

[Received: December 2, 2014, Accepted: January 16, 2015.]

\begin{abstract}
The present paper deals with the development of the two-dimensional $J$-integral for large strains. The line of thought is based on a continuum mechanical approach by using elastic or elastic-plastic bodies and presents some numerical examples.
\end{abstract}

\section{INTRODUCTION}

The phenomenon of failure caused by catastrophic crack propagation in structural materials poses problems of design and analysis in many fields of engineering. Cracks are present to some degree in all structures. They may exist as basic defects in the constituent materials or they may be induced in construction or during service life.

Using the finite element method, a lot of papers deal with the calculation of stress intensity factors for two- and three-dimensional models of solid bodies which contain cracks of different shapes and are subjected to various loading conditions. In order to increase the accuracy of the results, special singular and transition elements have been used. These are described together with the methods that are used to determine the stress intensity factors from the results computed. The methods mentioned include the displacement substitution method, $J$-integral and the virtual crack extension technique.

Over the past decades the finite element technique has become firmly established as a useful tool for numerical solution of engineering problems. In order to be able to apply the finite element method to the efficient solution of fracture problems, appropriate adaptations and/or further developments must be made.

At the vicinity of a crack tip the strains are not always small, sometimes they may be large ones, too. The $J$-integral can also be applied to characterize the cracks in elastic or elastic-plastic bodies under the assumption of finite strains.

In the literature there are only a few papers dealing with the $J$-integral for large strains. For example Lau at al. [1, 2] presented a revised J-estimation method under large plastic deformation. May and Kobayashi [3] investigated plane stress stable crack growth and $J$-integral using Moire interferometry. Boothman at al. [4] developed the $J$ - and $Q$-estimation schemes for homogeneous plates. Jackiewicz [5] applied a hybrid model of steel cracking. Bouchard at al. 6] demonstrated their 
two-dimensional local approach finite element study compared with conventional $J$ estimation schemes and cracked body $J$-integral analysis. Saczuk at al. 7 presented a continuum model with inelastic material behaviour and a generalization of the $J$ integral.

The aim of the present paper is a further development of the two-dimensional $J$ integral based on continuum mechanics assuming large strains and elastic or elasticplastic material behavior: computation of the $J$-integral is made by using the finite element method and the numerical results show the efficiency of the procedure we have developed.

\section{Fundamental Concepts and notations}

Continuum mechanics is the part of mechanics that deals with the mechanical motion of bodies using continuum models. The general theory of continuum mechanics applies to 3-dimensional models. It is, in general, supposed that the continuum (in the present case the solid body considered) has a deformation and stress-free initial state referred to as initial configuration for which $t=t_{0}=0$. At time $t$ (when we perform our investigation) the state of continuum is referred to as present configuration.

The mechanical motion of a continuum is analyzed in a reference coordinate system which is usually the cartesian coordinate system $(x y z)$. An arbitrary point of the moving continuum is denoted by $\widehat{P}$. The position of this point is $P^{o}$ in the initial configuration and $P$ in the present configuration. In the coordinate system $(x y z)$ the coordinates of the point $P^{o}$ are $x^{o}, y^{o}, z^{o}$, the corresponding base vectors are denoted by $\mathbf{e}_{x}^{o}, \mathbf{e}_{y}^{o}$ and $\mathbf{e}_{z}^{o}$. The coordinates of the point $P$ are $x, y, z$. For the sake of making a difference the base vectors in the present configuration are denoted by $\mathbf{e}_{x}, \mathbf{e}_{y}$ and $\mathbf{e}_{z}$ $\left[\mathbf{e}_{m}^{o}=\mathbf{e}_{m}(m=x, y, z)\right]$. In accordance with what has been said above the quantities in the initial configuration are designated by the superscript ${ }^{\circ}$.

Scalar quantities are typeset in mathematical italic letters, e.g. $U, s$. Boldface letters stand for vector quantities: e.g. u, E. Tensors of order two are denoted by slanted boldface letters, e.g. $\boldsymbol{T}, \boldsymbol{F}$.

When using indicial notation in the cartesian coordinate system (a) 1, 2 and 3 correspond to $x, y, z$; (b) all the indices are subscripts, (c) summation over repeated indices is implied. The other notational conventions are the same as before.

The inverse of a tensor is denoted by the superscript ${ }^{-1}$, the notation for a transposed tensor is the superscript ${ }^{T}$. Scaler and double scaler multiplications are denoted by a dot, or a double-dot (energy product of two tensors of the second order).

We remark that the Lagrangian description will be used throughout the present paper.

\section{DESCRIPTION OF THE $J$-INTEGRAL}

Figure 1 shows a line integral path which encloses the crack tip and has initial and end points which lie on the two crack faces. It has been shown independently by Rice [8] and Cherepanov [9] that the following integral quantity is path independent when taken along any path, which satisfies the above conditions: 


$$
J=\int_{\Gamma}\left(U n_{x}-T_{i} \frac{\partial u_{i}}{\partial x}\right) \mathrm{d} s
$$

In this formula $U$ is the strain energy density, $T_{i}$ is the traction vector on a plane defined by the outward normal, $n_{i}, u_{i}$ is the displacement vector, $\mathrm{d} s$ is the arc element along the path, $\Gamma$. For a closed path not containing the crack tip, $J=0$ [ $]$.

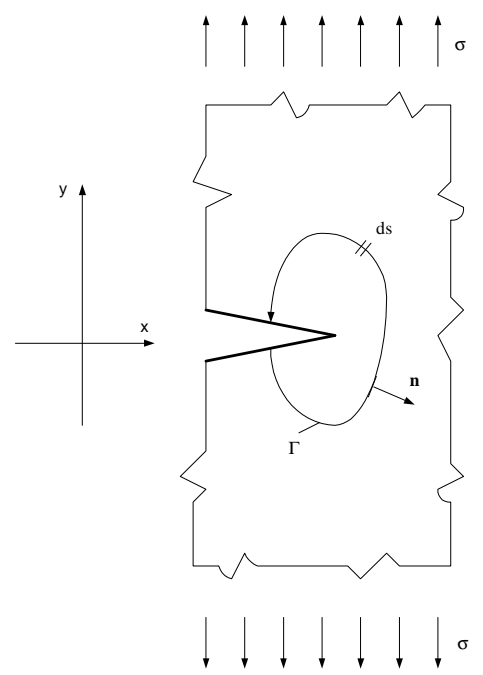

Figure 1. Contour path for $J$-integral evaluation

Knowles and Sternberg [10] noted that this expression could be considered as the first component of a vector:

$$
J_{k}=\int_{\Gamma}\left(U n_{k}-T_{i} \frac{\partial u_{i}}{\partial x_{k}}\right) \mathrm{d} s, \quad k=1,2 .
$$

This integral is also path independent provided that the contour touches each surface of the crack at the tip. As $x_{1}=x$ and $x_{2}=y$, applying formulae $n_{x}=\mathrm{d} y / \mathrm{d} s$ and $n_{y}=-\mathrm{d} x / \mathrm{d} s$, by means of 2 we can write the two components of the $J$-integral in the following form:

$$
J_{x}=\int_{\Gamma}\left(U \mathrm{~d} y-T_{i} \frac{\partial u_{i}}{\partial x} \mathrm{~d} s\right), \quad J_{y}=-\int_{\Gamma}\left(U \mathrm{~d} x+T_{i} \frac{\partial u_{i}}{\partial y}\right) .
$$

For elastic-plastic bodies the strain energy density consists of two parts:

$$
U=U_{e}+U_{p}
$$

$U_{e}$ is given by

$$
U_{e}=\frac{1}{2} \sigma_{i j}\left(\varepsilon_{i j}\right)_{e},
$$

where $\sigma_{i j}$ is the stress tensor and $\left(\varepsilon_{i j}\right)_{e}$ denotes the elastic components of strains. The plastic work contribution is given by 


$$
U_{p}=\int_{0}^{\bar{\varepsilon}_{p}} \bar{\sigma} \mathrm{d} \bar{\varepsilon}_{p}
$$

In this expression $\bar{\sigma}$ and $\bar{\varepsilon}_{p}$ are the effective stress and effective plastic strain, respectively:

$$
\bar{\sigma}=\left[\frac{3}{2}\left(\sigma_{i j}^{\prime} \sigma_{i j}^{\prime}\right)\right]^{1 / 2},
$$

in which $\sigma_{i j}^{\prime}$ denotes the components of the deviatoric stress tensor and

$$
\mathrm{d} \bar{\varepsilon}_{p}=\left\{\frac{2}{3}\left[\left(\mathrm{~d} \varepsilon_{i j}\right)_{p}\left(\mathrm{~d} \varepsilon_{i j}\right)_{p}\right]\right\}^{1 / 2}
$$

where $\left(\mathrm{d} \varepsilon_{i j}\right)_{p}$ denotes the plastic part of the strain tensor increment.

Figure 2 represents the motion of a continuum with the initial and the present configurations.

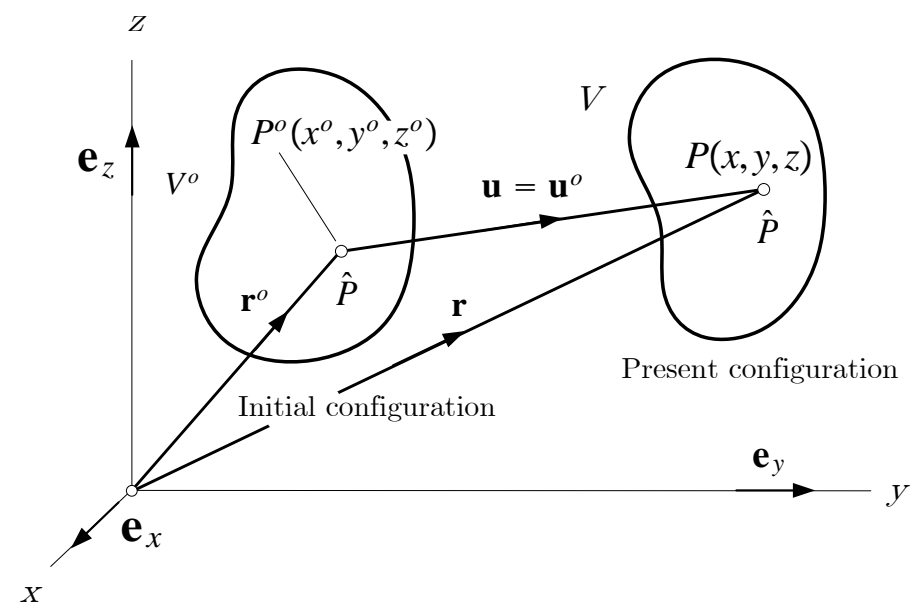

Figure 2. Motion of the continuum in the reference coordinate system $(x y z)$

Let us suppose that equation $(2)$ is valid in the present configuration for large strains. As the initial configuration is known, it is necessary to express the quantities in the integrand in terms of the Green-Lagrange strain tensor $\left(\boldsymbol{E}^{0}\right)$ and the second PiolaKirchhoff stress tensor $\left(\boldsymbol{T}^{0}\right)$. For elastic applications it can be proved that instead of the strain energy density $U$ one can use the following formula:

$$
U^{o}=\frac{1}{2} \boldsymbol{E}^{o} \cdot \boldsymbol{T}^{o}
$$

For two-dimensional problems equation (9) assumes the form:

$$
U^{o}=\frac{1}{2}\left(E_{x x}^{o} T_{x x}^{o}+2 E_{x y}^{o} T_{x y}^{o}+E_{y y}^{o} T_{y y}^{o}\right) .
$$

The arc element is

$$
\mathrm{d} s=\mathrm{d} s^{o} \lambda_{s}=\mathrm{d} s^{o} \sqrt{1+2 \mathbf{e}^{o} \cdot \boldsymbol{E}^{o} \cdot \mathbf{e}^{o}},
$$


where $\mathrm{d} s^{o}$ and $\mathbf{e}^{o}$ are the arc element and the tangent vector to the curve $\Gamma$ in the initial configuration and $\lambda_{s}$ is the stretch.

For manipulating the traction vector $\mathbf{t}=\boldsymbol{T} \cdot \mathbf{n}$ into a suitable form we shall need the relations:

$$
\begin{gathered}
\boldsymbol{T}=\frac{1}{\mathfrak{J}} \boldsymbol{F} \cdot \boldsymbol{T}^{o} \cdot \boldsymbol{F}^{T}, \\
\mathfrak{J}=\operatorname{det}|\boldsymbol{F}|,
\end{gathered}
$$

where $\boldsymbol{F}=\mathbf{r} \circ \nabla^{o}$ is the deformation gradient, $\mathfrak{J}$ is the Jacobian and $\nabla^{o}$ is the nabla operator in the initial configuration.

Applying the formula d $\mathbf{A}=\mathfrak{J} \boldsymbol{F}^{-T} \cdot \mathrm{d} \mathbf{A}^{o}$ between the surface elements, the traction vector can be expressed as:

$$
\mathbf{t}=\frac{1}{\lambda_{A}} \boldsymbol{F} \cdot \boldsymbol{T}^{o} \cdot \mathbf{n}^{o}=\frac{1}{\mathfrak{J} \sqrt{\mathbf{n}^{o} \cdot\left(2 \boldsymbol{E}^{o}+\boldsymbol{I}\right)^{-1} \cdot \mathbf{n}^{o}}} \boldsymbol{F} \cdot \boldsymbol{T}^{o} \cdot \mathbf{n}^{o},
$$

where $\boldsymbol{I}$ is the unit tensor, $\mathbf{n}^{o}$ is the outward unit normal to $\Gamma$ in the initial configuration, $\mathrm{d} \mathbf{A}$ is the vectorial surface element in the present configuration, $\mathrm{d} \mathbf{A}^{o}$ is the vectorial surface element in the initial configuration, $\lambda_{A}=\mathrm{d} A / \mathrm{d} A^{o}$ is the ratio of the scalar surface element [11.

It can be seen from Figure 2 that $\mathbf{r}=\mathbf{r}^{o}+\mathbf{u}^{o}$, therefore we can write

$$
\begin{aligned}
& \mathrm{d} y=\frac{\partial y}{\partial x^{o}} \mathrm{~d} x^{o}+\frac{\partial y}{\partial y^{o}} \mathrm{~d} y^{o}=\mathrm{d} y^{o}+\frac{\partial u_{y}^{o}}{\partial x^{o}} \mathrm{~d} x^{o}+\frac{\partial u_{y}^{o}}{\partial y^{o}} \mathrm{~d} y^{o} \\
& \mathrm{~d} x=\frac{\partial x}{\partial x^{o}} \mathrm{~d} x^{o}+\frac{\partial x}{\partial y^{o}} \mathrm{~d} y^{o}=\mathrm{d} x^{o}+\frac{\partial u_{x}^{o}}{\partial x^{o}} \mathrm{~d} x^{o}+\frac{\partial u_{x}^{o}}{\partial y^{o}} \mathrm{~d} y^{o} .
\end{aligned}
$$

As $\mathbf{u}=\mathbf{u}^{o}$, the derivatives of the displacement vector are as follows:

$$
\begin{gathered}
\frac{\partial \mathbf{u}^{o}}{\partial x}=\frac{\partial u_{x}^{o}}{\partial x} \mathbf{e}_{x}^{o}+\frac{\partial u_{y}^{o}}{\partial x} \mathbf{e}_{y}^{o}=\left(\frac{\partial u_{x}^{o}}{\partial x^{o}} \frac{\partial x^{o}}{\partial x}+\frac{\partial u_{x}^{o}}{\partial y^{o}} \frac{\partial y^{o}}{\partial x}\right) \mathbf{e}_{x}^{o}+\left(\frac{\partial u_{y}^{o}}{\partial x^{o}} \frac{\partial x^{o}}{\partial x}+\frac{\partial u_{y}^{o}}{\partial y^{o}} \frac{\partial y^{o}}{\partial x}\right) \mathbf{e}_{y}^{o}, \\
\frac{\partial \mathbf{u}^{o}}{\partial y}=\frac{\partial u_{x}^{o}}{\partial y} \mathbf{e}_{x}^{o}+\frac{\partial u_{y}^{o}}{\partial y} \mathbf{e}_{y}^{o}=\left(\frac{\partial u_{x}^{o}}{\partial x^{o}} \frac{\partial x^{o}}{\partial y}+\frac{\partial u_{x}^{o}}{\partial y^{o}} \frac{\partial y^{o}}{\partial y}\right) \mathbf{e}_{x}^{o}+\left(\frac{\partial u_{y}^{o}}{\partial x^{o}} \frac{\partial x^{o}}{\partial y}+\frac{\partial u_{y}^{o}}{\partial y^{o}} \frac{\partial y^{o}}{\partial y}\right) \mathbf{e}_{y}^{o} .
\end{gathered}
$$

Since $\mathbf{r}=\mathbf{r}^{o}+\mathbf{u}^{o}$, it follows that

$$
x=x^{o}+u_{x}^{o}, \quad y=y^{o}+u_{y}^{o} .
$$

Making use of 19 for the deformation gradient $\boldsymbol{F}$, the inverse deformation gradient $\boldsymbol{F}^{-1}$ and the Jacobian determinant $\mathfrak{J}$ we obtain

$$
\begin{gathered}
{[\boldsymbol{F}]=\left[\begin{array}{cc}
\frac{\partial x}{\partial x^{o}} & \frac{\partial x}{\partial y^{o}} \\
\frac{\partial y}{\partial x^{o}} & \frac{\partial y}{\partial y^{o}}
\end{array}\right]=\left[\begin{array}{cc}
1+\frac{\partial u_{x}^{o}}{\partial x^{o}} & \frac{\partial u_{x}^{o}}{\partial y^{o}} \\
\frac{\partial u_{y}^{o}}{\partial x^{o}} & 1+\frac{\partial u_{y}^{o}}{\partial y^{o}}
\end{array}\right],} \\
{\left[\boldsymbol{F}^{-1}\right]=\left[\begin{array}{cc}
\frac{\partial x^{o}}{\partial x} & \frac{\partial x^{o}}{\partial y} \\
\frac{\partial y^{o}}{\partial x} & \frac{\partial y^{o}}{\partial y}
\end{array}\right]=\frac{1}{\mathfrak{J}}\left[\begin{array}{cc}
1+\frac{\partial u_{y}^{o}}{\partial y^{o}} & -\frac{\partial u_{x}^{o}}{\partial y^{o}} \\
-\frac{\partial u_{y}^{o}}{\partial x^{o}} & 1+\frac{\partial u_{x}^{o}}{\partial x^{o}}
\end{array}\right],}
\end{gathered}
$$




$$
\mathfrak{J}=\operatorname{det}|\boldsymbol{F}|=\left(1+\frac{\partial u_{x}^{o}}{\partial x^{o}}\right)\left(1+\frac{\partial u_{y}^{o}}{\partial y^{o}}\right)-\frac{\partial u_{x}^{o}}{\partial y^{o}} \frac{\partial u_{y}^{o}}{\partial x^{o}} .
$$

Utilizing equations (20), (21) and $(22$, we can rewrite equations (17) and 18 in other form:

$$
\begin{aligned}
& \frac{\partial \mathbf{u}^{o}}{\partial x}=\frac{1}{\mathfrak{J}}\left[\left(1+\frac{\partial u_{y}^{o}}{\partial y^{o}}\right) \frac{\partial u_{x}^{o}}{\partial x^{o}}-\frac{\partial u_{x}^{o}}{\partial y^{o}} \frac{\partial u_{y}^{o}}{\partial x^{o}}\right] \mathbf{e}_{x}^{o}+\frac{1}{\mathfrak{J}}\left[\left(1+\frac{\partial u_{y}^{o}}{\partial y^{o}}\right) \frac{\partial u_{y}^{o}}{\partial x^{o}}-\frac{\partial u_{y}^{o}}{\partial y^{o}} \frac{\partial u_{y}^{o}}{\partial x^{o}}\right] \begin{array}{c}
\mathbf{e}_{y}^{o}, \\
(23)
\end{array} \\
& \frac{\partial \mathbf{u}^{o}}{\partial y}=\frac{1}{\mathfrak{J}}\left[\left(1+\frac{\partial u_{x}^{o}}{\partial x^{o}}\right) \frac{\partial u_{x}^{o}}{\partial y^{o}}-\frac{\partial u_{x}^{o}}{\partial x^{o}} \frac{\partial u_{x}^{o}}{\partial y^{o}}\right] \mathbf{e}_{x}^{o}+\frac{1}{\mathfrak{J}}\left[\left(1+\frac{\partial u_{x}^{o}}{\partial x^{o}}\right) \frac{\partial u_{y}^{o}}{\partial y^{o}}-\frac{\partial u_{y}^{o}}{\partial x^{o}} \frac{\partial u_{x}^{o}}{\partial y^{o}}\right] \mathbf{e}_{y}^{o} .
\end{aligned}
$$

Substituting (10) - 24 into (3) we obtain the components of $J$-integral for large strains in two dimensions:

$$
\begin{gathered}
J_{x}=\int_{(\Gamma)}\left[U^{o}\left(\mathrm{~d} y^{o}+\frac{\partial u_{y}^{o}}{\partial x^{o}} \mathrm{~d} x^{o}+\frac{\partial u_{y}^{o}}{\partial y^{o}} \mathrm{~d} y^{o}\right)-\mathbf{t} \frac{\partial \mathbf{u}^{o}}{\partial x} \lambda_{s} \mathrm{~d} s^{o}\right] \\
J_{y}=\int_{(\Gamma)}\left[-U^{o}\left(\mathrm{~d} x^{o}+\frac{\partial u_{x}^{o}}{\partial x^{o}} \mathrm{~d} x^{o}+\frac{\partial u_{x}^{o}}{\partial y^{o}} \mathrm{~d} y^{o}\right)-\mathbf{t} \frac{\partial \mathbf{u}^{o}}{\partial y} \lambda_{s} \mathrm{~d} s^{o}\right]
\end{gathered}
$$

For elastic-plastic bodies the strain energy density also has two parts:

$$
U^{o}=U_{e}^{o}+U_{p}^{o}
$$

where $U_{e}^{o}$ is given in equation $(9)$ and $U_{p}^{o}$ is similar to (6):

$$
U_{p}^{o}=\int_{o}^{\bar{E}_{p}^{o}} \bar{T}^{o} \mathrm{~d} \bar{E}_{p}^{o} .
$$

In this expression $\bar{T}^{o}$ and $\bar{E}_{p}^{o}$ are the effective stress and effective plastic strain in the initial configuration.

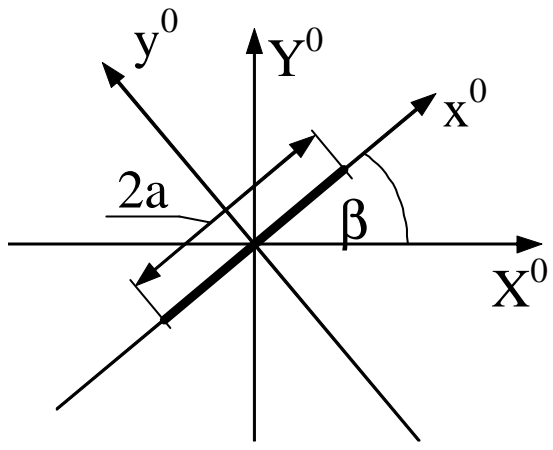

Figure 3. Coordinate systems in the initial configuration

For inclined cracks two coordinate systems and the corresponding transformation formulae are necessary - see Figure 3 . 
When one applies the finite element method the integration in equations 25 and (26) should be performed numerically.

\section{Path-independence of the $J$-integral}

Rice has already investigated the problem of path-independence [8]. We remark that other researchers have also examined this question, e.g. Atluri 12], Brocks and Scheider [13] and Wang at al. [14].

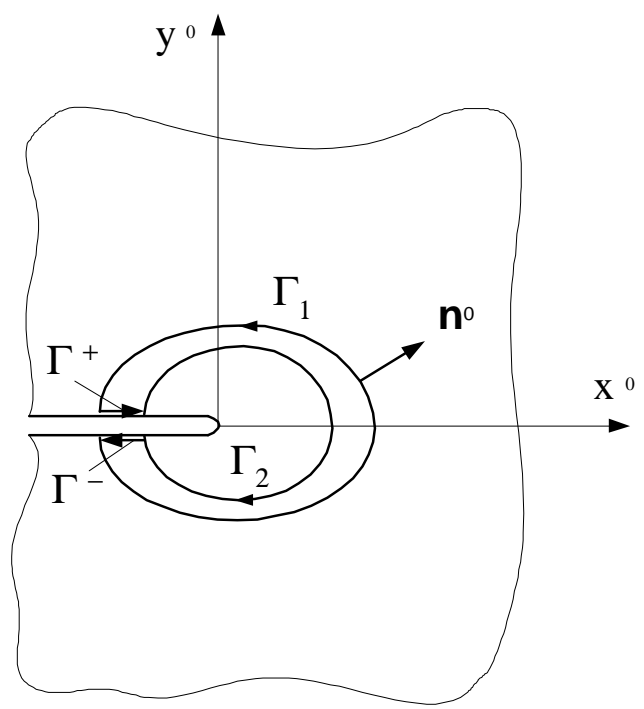

Figure 4. Closed contour for $J$-integral evaluation

Henceforth the path-independence of 25, 26) is proved for large strains and two dimensional problems. The following assumptions have been made:

- The material of the body is homogeneous.

- There are no body forces.

- The stress and strain fields depend on two coordinates $\left(x^{o}, y^{o}\right)$.

- The crack is straight.

- The stress-free crack borders are parallel to coordinate axis $x^{o}$.

Figure 4 shows a closed integral path which does not contain the crack tip.

The closed contour $\Gamma$ does not include a singularity:

$$
\Gamma=\Gamma_{1} \cup \Gamma^{+} \cup \Gamma_{2} \cup \Gamma^{-} .
$$

Then $J=0$ along a closed contour $\Gamma$ for large strains, too. Let us examine the component $J_{x}$ along the path $\Gamma$.

$$
J_{x}=0=\oint_{\Gamma_{2}}\left[U^{o}\left(\mathrm{~d} y^{o}+\frac{\partial u_{y}^{o}}{\partial x^{o}} \mathrm{~d} x^{o}+\frac{\partial u_{y}^{o}}{\partial y^{o}} \mathrm{~d} y^{o}\right)-\mathbf{t} \cdot \frac{\partial \mathbf{u}^{o}}{\partial x} \lambda_{s} \mathrm{~d} s^{o}\right]+
$$




$$
\begin{aligned}
& +\oint_{\Gamma_{1}}\left[U^{o}\left(\mathrm{~d} y^{o}+\frac{\partial u_{y}^{o}}{\partial x^{o}} \mathrm{~d} x^{o}+\frac{\partial u_{y}^{o}}{\partial y^{o}} \mathrm{~d} y^{o}\right)-\mathbf{t} \cdot \frac{\partial \mathbf{u}^{o}}{\partial x} \lambda_{s} \mathrm{~d} s^{o}\right]+ \\
& +\int_{\Gamma^{+}}\left[U^{o}\left(\mathrm{~d} y^{o}+\frac{\partial u_{y}^{o}}{\partial x^{o}} \mathrm{~d} x^{o}+\frac{\partial u_{y}^{o}}{\partial y^{o}} \mathrm{~d} y^{o}\right)-\mathbf{t} \cdot \frac{\partial \mathbf{u}^{o}}{\partial x} \lambda_{s} \mathrm{~d} s^{o}\right]+ \\
& +\int_{\Gamma^{-}}\left[U^{o}\left(\mathrm{~d} y^{o}+\frac{\partial u_{y}^{o}}{\partial x^{o}} \mathrm{~d} x^{o}+\frac{\partial u_{y}^{o}}{\partial y^{o}} \mathrm{~d} y^{o}\right)-\mathbf{t} \cdot \frac{\partial \mathbf{u}^{o}}{\partial x} \lambda_{s} \mathrm{~d} s^{o}\right]
\end{aligned}
$$

The integrals on $\Gamma^{+}$and $\Gamma^{-}$differ from each other in sign only. Therefore they can be dropped. In this way we get

$$
\begin{aligned}
J_{x}=0 & =\oint_{\Gamma_{2}}\left[U^{o}\left(\mathrm{~d} y^{o}+\frac{\partial u_{y}^{o}}{\partial x^{o}} \mathrm{~d} x^{o}+\frac{\partial u_{y}^{o}}{\partial y^{o}} \mathrm{~d} y^{o}\right)-\mathbf{t} \cdot \frac{\partial \mathbf{u}^{o}}{\partial x} \lambda_{s} \mathrm{~d} s^{o}\right]+ \\
& +\oint_{\Gamma_{1}}\left[U^{o}\left(\mathrm{~d} y^{o}+\frac{\partial u_{y}^{o}}{\partial x^{o}} \mathrm{~d} x^{o}+\frac{\partial u_{y}^{o}}{\partial y^{o}} \mathrm{~d} y^{o}\right)-\mathbf{t} \cdot \frac{\partial \mathbf{u}^{o}}{\partial x} \lambda_{s} \mathrm{~d} s^{o}\right] .
\end{aligned}
$$

We get a useful expression if the integration on contour $\Gamma_{2}$ is performed counterclockwise:

$$
\begin{aligned}
& \oint_{\Gamma_{2}}\left[U^{o}\left(\mathrm{~d} y^{o}+\frac{\partial u_{y}^{o}}{\partial x^{o}} \mathrm{~d} x^{o}+\frac{\partial u_{y}^{o}}{\partial y^{o}} \mathrm{~d} y^{o}\right)-\mathbf{t} \cdot \frac{\partial \mathbf{u}^{o}}{\partial x} \lambda_{s} \mathrm{~d} s^{o}\right]= \\
= & -\oint_{\Gamma_{2}}\left[U^{o}\left(\mathrm{~d} y^{o}+\frac{\partial u_{y}^{o}}{\partial x^{o}} \mathrm{~d} x^{o}+\frac{\partial u_{y}^{o}}{\partial y^{o}} \mathrm{~d} y^{o}\right)-\mathbf{t} \cdot \frac{\partial \mathbf{u}^{o}}{\partial x} \lambda_{s} \mathrm{~d} s^{o}\right] .
\end{aligned}
$$

Substituting (32) into equation (31) we obtain the following formula:

$$
\begin{aligned}
0= & -\oint_{\Gamma_{2}}\left[U^{o}\left(\mathrm{~d} y^{o}+\frac{\partial u_{y}^{o}}{\partial x^{o}} \mathrm{~d} x^{o}+\frac{\partial u_{y}^{o}}{\partial y^{o}} \mathrm{~d} y^{o}\right)-\mathbf{t} \cdot \frac{\partial \mathbf{u}^{o}}{\partial x} \lambda_{s} \mathrm{~d} s^{o}\right]+ \\
& +\oint_{\Gamma_{1}}\left[U^{o}\left(\mathrm{~d} y^{o}+\frac{\partial u_{y}^{o}}{\partial x^{o}} \mathrm{~d} x^{o}+\frac{\partial u_{y}^{o}}{\partial y^{o}} \mathrm{~d} y^{o}\right)-\mathbf{t} \cdot \frac{\partial \mathbf{u}^{o}}{\partial x} \lambda_{s} \mathrm{~d} s^{o}\right]
\end{aligned}
$$

Rearrangement of (34) results in

$$
\begin{aligned}
& \oint_{\Gamma_{2}}\left[U^{o}\left(\mathrm{~d} y^{o}+\frac{\partial u_{y}^{o}}{\partial x^{o}} \mathrm{~d} x^{o}+\frac{\partial u_{y}^{o}}{\partial y^{o}} \mathrm{~d} y^{o}\right)-\mathbf{t} \cdot \frac{\partial \mathbf{u}^{o}}{\partial x} \lambda_{s} \mathrm{~d} s^{o}\right]=+ \\
=+ & \oint_{\Gamma_{1}}\left[U^{o}\left(\mathrm{~d} y^{o}+\frac{\partial u_{y}^{o}}{\partial x^{o}} \mathrm{~d} x^{o}+\frac{\partial u_{y}^{o}}{\partial y^{o}} \mathrm{~d} y^{o}\right)-\mathbf{t} \cdot \frac{\partial \mathbf{u}^{o}}{\partial x} \lambda_{s} \mathrm{~d} s^{o}\right] .
\end{aligned}
$$

which shows the path independence of the first component of the vector $J$. This holds for the other component, too.

\section{ApplicABility of SPECIAL isoparametriC ElEMENTS}

Consider a one-dimensional element that may form a side of a $2 \mathrm{D}$ or $3 \mathrm{D} n$ th-order isoparametric element (see Figure 5). 

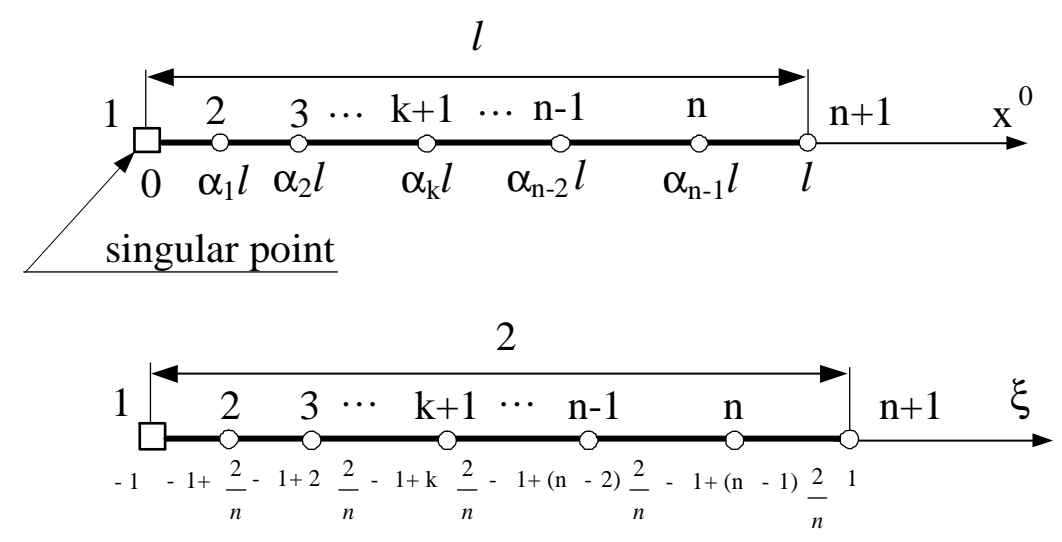

Figure 5. Element coordinate mapping

The above transformation is accomplished by means of the usual isoparametric mapping technique [15]. Without entering into details we obtain the following expressions:

$$
\begin{gathered}
x^{o}=\frac{\ell}{2^{m}}(1+\xi)^{m}, \\
\xi=-1+2\left(\frac{x^{o}}{\ell}\right)^{1 / m} .
\end{gathered}
$$

For an isoparametric element the displacement $u_{x}^{o}$ takes the form

$$
u_{x}^{o}=b_{0}+b_{1} \xi+b_{2} \xi^{2}+\cdots+b_{n} \xi^{n}, \quad n \geqq 2 .
$$

from where

$$
\frac{\partial u_{x}^{o}}{\partial \xi}=b_{1}+2 b_{2} \xi+3 b_{3} \xi^{2}+\cdots+n b_{n} \xi^{(n-1)} .
$$

This expression can be manipulated further by inserting (36)

$$
\begin{aligned}
\frac{\partial u_{x}^{o}}{\partial \xi} & =b_{1}+2 b_{2}\left[-1+2\left(\frac{x^{o}}{\ell}\right)^{1 / m}\right]+3 b_{3}\left[-1+2\left(\frac{x^{o}}{\ell}\right)^{1 / m}\right]^{2}+\cdots+ \\
& +n b_{n}\left[-1+2\left(\frac{x^{o}}{\ell}\right)^{1 / m}\right]^{(n-1)} .
\end{aligned}
$$

The strain in the $x^{o}$-direction is then

$$
E_{x x}^{o}=\frac{\mathrm{d} u_{x}^{o}}{\mathrm{~d} x^{o}}+\frac{1}{2}\left(\frac{\mathrm{d} u_{x}^{o}}{\mathrm{~d} x^{o}}\right)^{2} .
$$

Utilizing equation (37) the axial strain 40 can be rewritten

$$
E_{x x}^{o}=\frac{\mathrm{d} u_{x}^{o}}{\mathrm{~d} \xi} \frac{\mathrm{d} \xi}{\mathrm{d} x^{o}}+\frac{1}{2}\left(\frac{\mathrm{d} u_{x}^{o}}{\mathrm{~d} \xi} \frac{\mathrm{d} \xi}{\mathrm{d} x^{o}}\right)^{2}
$$


Substituting (39) and the derivative of (36) into 41) we obtain

$$
\begin{aligned}
E_{x x}^{o} & =A_{1}\left(x^{o}\right)^{\frac{1-m}{m}}+A_{2}\left(x^{o}\right)^{\frac{2-m}{m}}+A_{3}\left(x^{o}\right)^{\frac{3-m}{m}}+\cdots+ \\
& +A_{n}\left(x^{o}\right)^{\frac{n-m}{m}}+A_{n+1}\left(x^{o}\right)^{\frac{2(1-m)}{m}}+A_{n+2}\left(x^{o}\right)^{\frac{2(1-m)+1}{m}}+\cdots+ \\
& +A_{(3 n-1)}\left(x^{o}\right)^{\frac{2(n-m)}{m}},
\end{aligned}
$$

where

$$
\begin{aligned}
A_{1} & =C\left(b_{1}-2 b_{2}+3 b_{3}-\cdots \pm n b_{n}\right), \\
A_{2} & =\frac{C}{\ell^{1 / m}} 2\left[2 b_{2}-6 b_{3}+12 b_{4}-\cdots \pm(n-1) n b_{n}\right], \\
& \vdots \\
A_{n} & =\frac{C}{\ell^{(n-1) / m}} 2^{(n-1)} n b_{n}, \\
A_{(n+1)} & =\frac{C^{2}}{2}\left[b_{1}^{2}+4 b_{2}^{2}+\cdots+n^{2} b_{n}^{2}-4 b_{1} b_{2}+6 b_{1} b_{3}-\cdots \pm 2 n b_{1} b_{n} \pm \cdots \pm\right. \\
& \left. \pm 2(n-1) n b_{(n-1)} b_{n}\right] \\
A_{(n+2)} & =\frac{C^{2}}{2} \frac{2^{2}}{\ell^{1 / m}}\left[2 b_{1} b_{2}-6 b_{1} b_{3}+18 b_{2} b_{3}-4 b_{2}^{2}-18 b_{3}^{2}-\cdots \pm\right. \\
& \left. \pm n^{2}(n-1) b_{(n-1)} b_{n}-(n-1)\left(n b_{n}\right)^{2}\right], \\
& \vdots \\
A_{(3 n-1)} & =\frac{C^{2}}{2} \frac{2^{2(n-1)}}{\ell^{2(n-1) / m}}\left(n b_{n}\right)^{2}, \\
C & =\frac{2}{m} \frac{1}{\ell^{1 / m}} \cdot
\end{aligned}
$$

Equation 42 clearly shows that the strain is singular at $x^{o}=0(\xi=-1)$. The leading strain term is of order $\left(x^{o}\right)^{\frac{1-m}{m}}$. Therefore when $x^{o} \rightarrow 0$, the type of the strain singularity is $\left(x^{o}\right)^{(1-m) / m}(m \geq 2)$.

\section{Numerical EXAmple}

The author has developed a Fortran program by means of Microsoft Developer Studio 97 to compute the $J$-integral numerically for small and large strains. For a real physical problem the strains can be either small or large depending on the loading of the body. As regards the present example both kind of strains are computed so that one can see what the difference is between the two kind of strains. When computing elastic-plastic problems the Von Mises yield criterion, the Newton-Raphson iteration technics and the Euler-Cauchy incremental method are applied. 


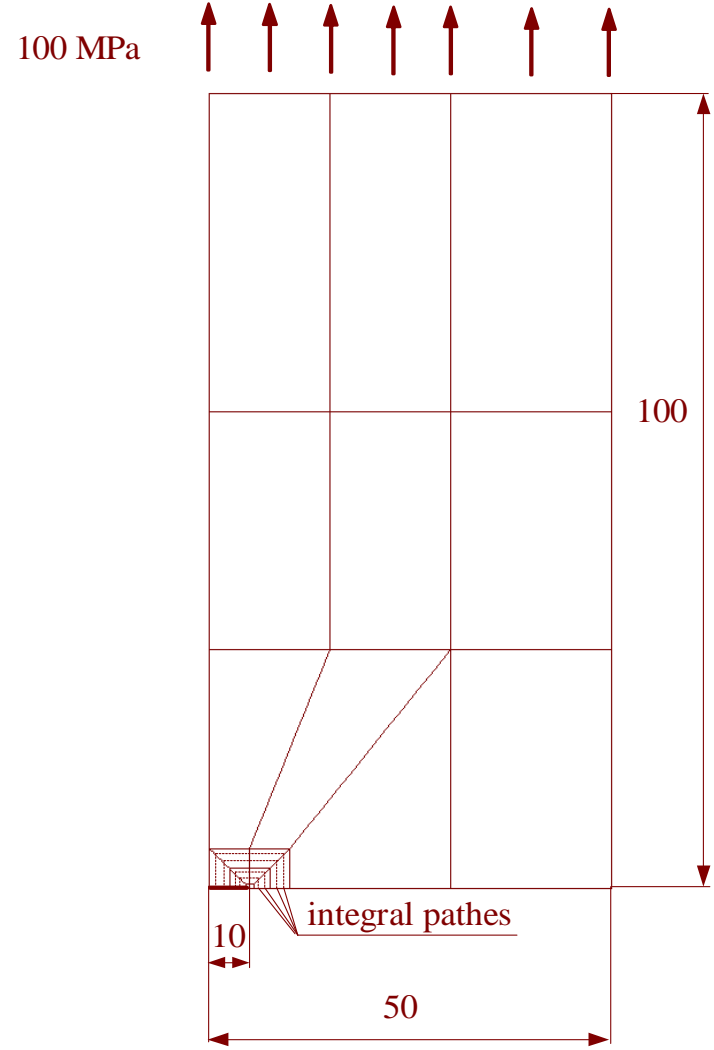

Figure 6. Finite element mesh

The example considered is that of a plate under tension which contains a crack of length $20 \mathrm{~mm}$ perpendicular to the direction of loading. The width of the plate is $100 \mathrm{~mm}$ and the thickness is assumed to be unity. The length of the plate is 200 $\mathrm{mm}$. For the first computations the material is linear elastic with the properties $E=2 \times 10^{5} \mathrm{MPa}$ and $\nu=0.3$. The applied tensile traction is $p=100 \mathrm{MPa}$. Because of the symmetrical properties of the problem the finite element mesh represents only a quarter of the body considered - see Figure 6 . The finite element mesh contains singular and transition elements as well.

Theoretically $J_{y}$ is zero for this problem. Figure 7 shows the values computed for the $J$-integral.

For the second series of computations the material of the plate is a linear elastic linear hardening material for which $H^{\prime}=0,1 E$ and the yield stress is $\sigma_{F}=100$ $\mathrm{MPa}$. The loading is applied in incremental steps. The increments are $0.1 p=10$ $\mathrm{MPa}, 1.0 p=100 \mathrm{MPa}, 0.3 p=30 \mathrm{MPa}$. Figure 8 shows the values computed for the $J$-integral both for small strains and for large strains.

Figure 9 depicts the plastic zones for the third load increment. Figure 10 shows the von Mises stresses for the third load increment. 


\section{J-integral}

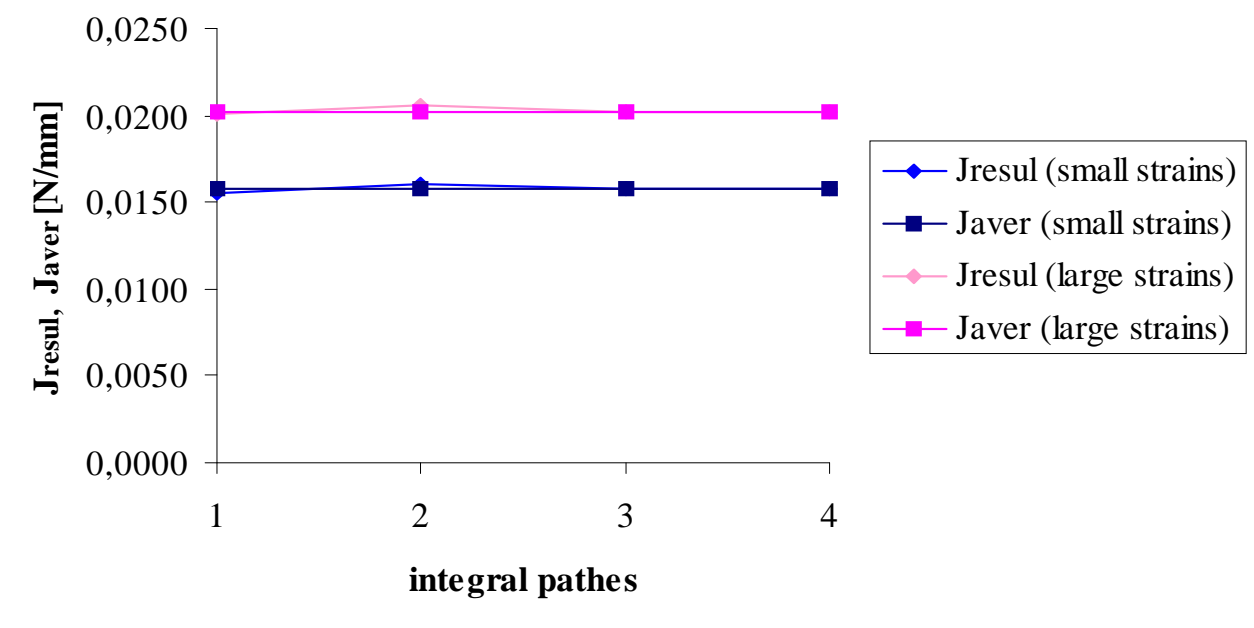

Figure 7. $J$-integral for elastic deformations

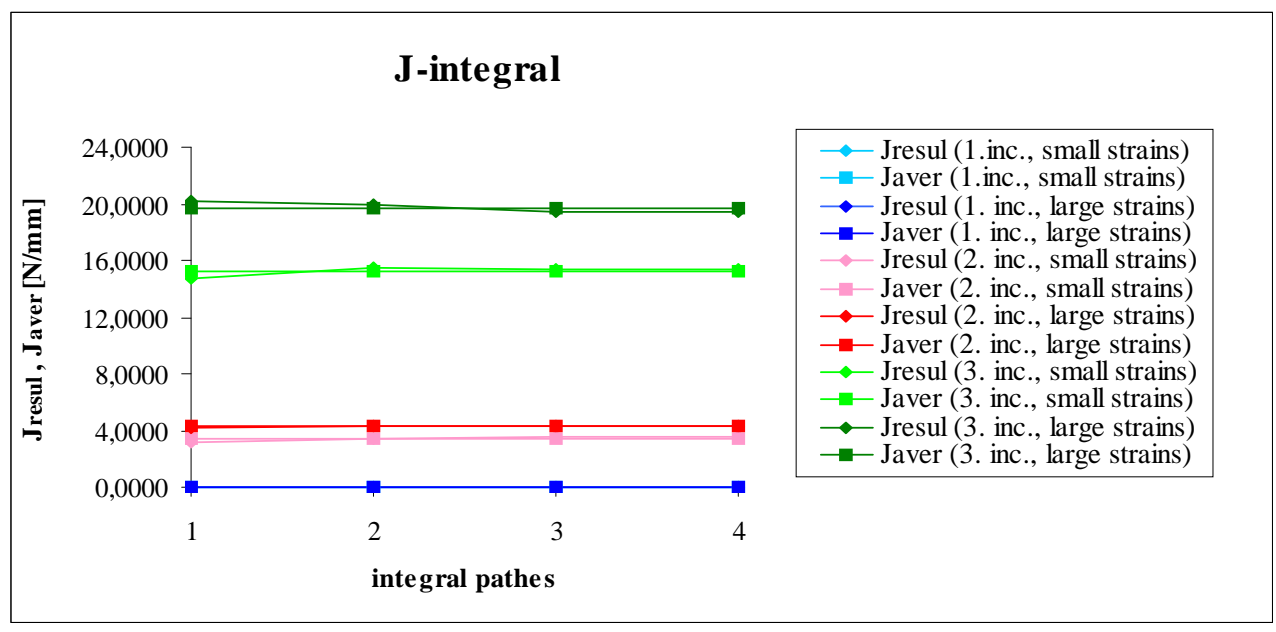

Figure 8. J-integral for elastic-plastic deformations 
Small strains

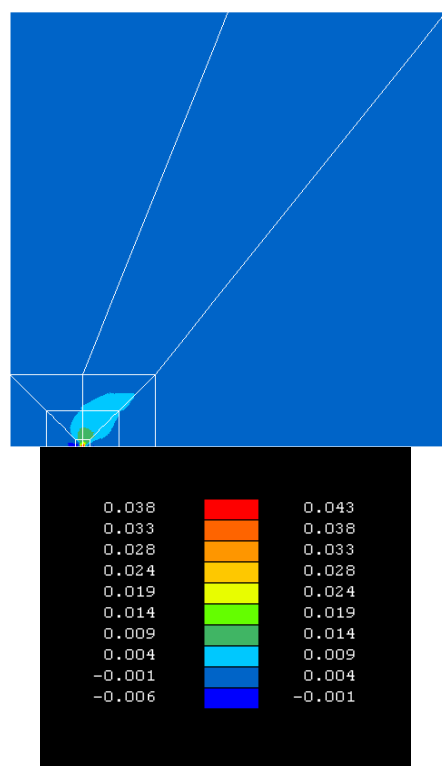

Large strains

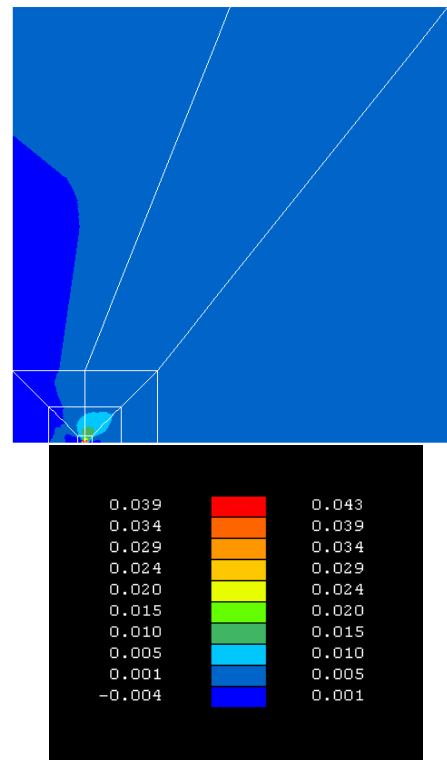

Figure 9. Plastic zones for the third load increment

Small strains
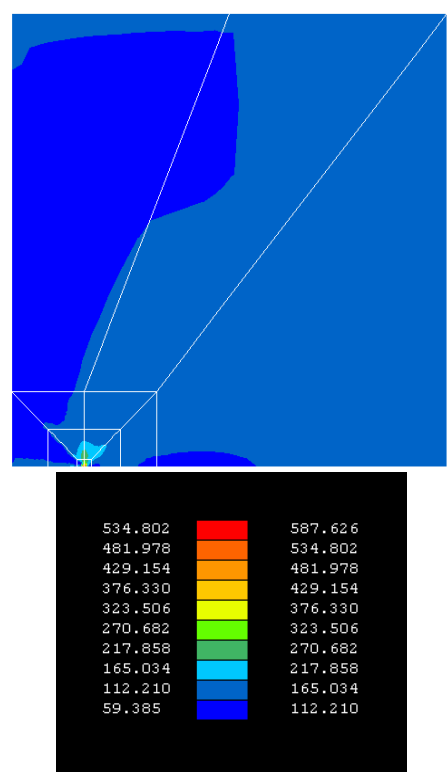

Large strains
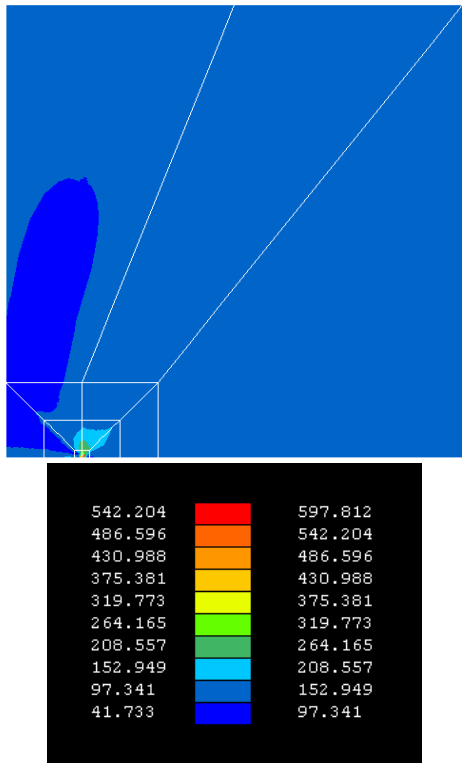

Figure 10. Von Mises stresses for the third load increment 


\section{Conclusions}

This paper proves the formulations and applicability of the $J$-integral for large strains under the assumption of elastic and elastic-plastic material behaviour. The $J$ integral values are higher for large strains, as can be seen from the numerical examples we have solved. This fact means that the safety of the cracked body increases from the aspect of service life. The path independence of the two-dimensional $J$-integral is also proved for large strains - the numerical results confirm the validity of this statement. The paper presents the mapping and applicability of special isoparametric elements for the finite element meshes. Using these elements the type of the strain singularity is $\left(x^{0}\right)^{(1-m) / m}(m \geq 2)$ at the crack tip. The application of the special isoparametric elements gives good results for relatively coarse finite element meshes, too.

\section{REFERENCES}

1. Lau, C. L., Lee, M. M. K., and Luxmoore, A. R.: Methodologies for Predicting jintegrals under Large Plastic Deformation-i. further Developments for Tension Loading. Eng. Frac. Mech., 49(3), (1994), 337-354.

2. Lau, C. L., Lee, M. M. K., and Luxmoore, A. R.: Methodologies for Predicting j-integrals under Large Plastic Deformation-ii. single Edge Notch Specimens in Pure Bending. Eng. Frac. Mech., 49(3), (1994), 355-369.

3. May, G. B. and Kobayashi, A. S.: Plane Stress Stable Crack Growth and Jintegral/hrr Field. Int. J. of Solids and Structures, 32(6/7), (1995), 857-881.

4. Boothman, D. P., Lee, M. M. K., and Luxmoore, A. R.: The Effects of Weld Mismatch on J-integrals and Q-values for Semi-elliptical Surface Flaws. Eng. Frac. Mech., 64, (1994), 433-458.

5. Jaczkiewicz, J.: Numerical Aspects of Non-local Modelling of the Damage Evolution in Elastic-plastic Materials. Comp. Mat. Science, 19, (2000), 235-251.

6. Bouchard, P. J., Goldthorpe, M. R., and Prottey, P.: J-integral and Local Damage Fracture Analyses for a Pump Casing Containing Large Weld Repairs. Int. J. Pressure Vessels, 78, (2001), 295-305.

7. Saczuk, J., Stumpf, H., and Vallee, C.: A Continuum Model Accounting for Defect and Mass Densities in Solids with Inelastic Material Behaviour. Int. J. of Solids and Structures, 38(52), (2001), 9545-9568.

8. RICE, J. R.: A Path Independent Integral and the Approximate Analysis of Strain Concentration by Notches and Cracks. J. App. Mech., 34, (1968), 379-386.

9. Cherepanov, G. P.: Cracks in Solids. Prikl. Mat. Mekh., 25, (1967), 476-488.

10. Knowles, J. K. and Sternberg, E.: On a Class of Conservation Laws in Linearized and Finite Elastostatics. Arch. Rat. Mech. Anal., 44, (1972), 187-211.

11. Béda, G., KozÁk, I., and Verhás, J.: Continuum Mechanics. Akadémiai Kiadó, Budapest, 1995.

12. AtLuRI, S. N.: Path-independent integrals in finite elasticity and inelasticity, with body forces, inertia, and arbitrary crack-face conditions. Eng. Frac. Mech., 16(3), (1982), 341364. 
13. Brocks, W. and Scheider, I.: Numerical Aspects of the Path-Dependence of the J-Integral in Incremental Plasticity. GKSS Forschungszentrum, Geesthacht, 1, (2001), $1-33$.

14. Wang, X.-M. and Shen, Y.-P.: The conservation laws and path-independent integrals with an application for linear electro-magneto-elastic media. Int. J. Solids Structures, 33(6), (1996), 865-878.

15. HorvÁtн, Á.: Higher-order Singular Isoparametric Elements for Crack Problems. Comm. in Num. Meth. Eng., 10, (1994), 73-80. 